\section{Régis Costello \\ Patrick Lecine \\ Brigitte Kahn-Perlès \\ Michèle Algarté \\ Carol Lipcey \\ Daniel Olive Jean Imbert}

\title{
Activation du système de facteurs de transcription Rel/NF-KB
}

Grâce à leurs propriétés d'activation et de modularité, les facteurs de transcription Rel/NF- $\kappa B$ constituent un élément majeur de la mise en place immédiate des réactions de défense de l'organisme. Ils participent à l'activation de nombreux gènes viraux et cellulaires impliqués en particulier dans la réponse immunitaire, tels que ceux codant pour des cytokines et leurs récepteurs. Si une activité basale de liaison à l'ADN est détectée dans le noyau de certaines cellules (lymphocyte B différencié et monocyte), le transport au noyau des facteurs Rel/NF-KB à partir de réserves cytoplasmiques inactives est induit dans la plupart des types cellulaires en réponse à de très nombreux stimuli: hormones, facteurs de croissance et cytokines, infections virales et bactériennes, stress. Cette redistribution nucléaire implique de multiples mécanismes, mutuellement non exclusifs, qui permettent une régulation fine de l'activation du système Rel/NF- $\kappa B$, adaptée à la fois au stimulus et au type cellulaire.

\begin{abstract}
ADRESSE
R. Costello: poste d'accueil Inserm. P. I.ecine: allocataire de recherche MESR. B. Kahn-Perles: chargée de recherche au Cinrs. M. Algarté: allocalaire de recherche MISSR. C. Lipcey : ingenieur a l'Inserm. D. Olive : directeur de recherche a l'Insenn. J. Imbert: chargé de recherche a $1 \% n$ sern. I aboratoire d'immunologie fonctionnelle et moléculaire, Inserm U. 1 19, 27, boulevard lei-Roure, 13009 Marseille, France.
\end{abstract}

Voir aussi la mini-synthèse d'A. Isrä̈l, page 1017 de ce numéro.

$\mathrm{m} / \mathrm{s} u^{\circ} 7$. vol. 11 , juillet 95 e système de facteurs de transcription Rel/NF-KI3 est un élément clé des mécanismes de réponse immédiate qui permettent à la cellule vivante d'adapter rapidement son programme d'expression génique à une situation nouvelle. I a caractéristique majeure de ce système dans de très nombreux types cellulaires est la possibilité, sous l'effet de stimuli variés (hormones, facteurs de croissance, cytokines, infections virale et bactérienne, lipopolysaccharides, esters de phorbol, radicaux oxygène libres, rayonnement ultraviolet...), de mobiliser rapidement (quelques minutes) des réserves cytoplasmiques de facteurs de transcription inactifs vers le noyau ( $n / s n^{\circ} 8$, vol. $6, p$. 803).
Cette activation nucléaire est constitutive dans un petit nombre de cellules différenciées comme les cellules $B$ différenciées, les monocytes et les neurones. La forme cytoplasmique la plus abondante du complexe NF-KB est un hétérotrimère inactif composé des sous-unités p50 $0^{N / \kappa \beta l}$, p $65^{\text {rels }}$ et de l'inhibiteur I I $B \alpha$. Le complexe p50/p65 est retenu dans le cytoplasme par son association à la sous-unité inhibitrice qui agit, notamment, en masquant les signaux de localisation nucléaire (NLS) des sous-unités. Lee stimulus activateur libère l'hétérodimère p50/p65 qui peut alors pénétrer dans le noyau, se fixer à l'ADN sur son élément régulateur spécifique $(\kappa B)$ et activer les gènes cibles qui le contiennent (Sigure 1). Les 


\section{RÉFÉRENCES}

1. Israēl A. Les protéines NF-кB, Dorsal et Rel, une nouvelle classe de facteurs de transcription. médecine/sciences 1991; 7:6770.

2. Siebenlist U, Franzoso G, Brown K. Structure, regulation and function of $\mathrm{NF}-\mathrm{KB}$. Annu Rev Cell Biol 1994; 10 : 405-55.

3. Shirakawa $\mathrm{F}$, Mizel SB. In vitro activation and nuclear translocation of NF- $K B$ catalyzed by cyclic AMP-dependent protein kinase and protein kinase C. Mol Cell Biol 1989; 9: 2424-30.

4. Ghosh S, Baltimore D. Activation in vitro of NF-KB by phosphorylation of its inhibitor IкB. Nature $1990 ; 344: 678-82$.

5. Thanos D, Maniatis T. NF-kappa B : a lesson in family value. Cell $1995 ; 80: 529-32$.

6. Chedid M, Mizel SB. Involvement of cyclic AMP-dependent protein kinases in the signal transduction pathway for interleukin1. Mol Cell Biol 1990; 10: 3824-7.

7. Chen D, Rothenberg EV. Interleukin 2 transcription factors as molecular targets of cAMP inhibition: delayed inhibition kinetics and combinatorial transcription roles. I Exp Med 1994; 179: 931-42.

8. Diaz-Meco MT, Berra E, Municio MM, Sanz L, Lozano J, et al. (13 auteurs). A dominant negative protein kinase $C \zeta$ subspecies blocks NF-KB activation. Mol Cell Biol $1993 ; 13: 4770-5$

9. Guery JC, Adorini L. Dendritic cells are the most efficient in presenting endogenous naturally processed self-epitopes to class II-restricted T cells. I Immunol 1995; 154: $536-44$

10. Gillepsie SKH, Wasserman SA. Dorsal, a drosophila Rel-like protein, is phosphorylated upon activation of the transmembrane protein Toll. Mol Cell Biol 1994; 14 : 3559-68.

11. Schreck R, Albermann K, Baeuerle PA. Nuclear factor kappa B : an oxidative stressresponsive transcription factor of eukaryotic cells. Free Rad Res Commun 1992; 17: 221-37.

12. Toledano MB, I.eonard WI. Modulation of transcription factor NF- $\mathrm{KB}$ binding activity by oxidation-reduction in vitro. Proc Nat Acad Sci USA 1991 ; 88: 4328-32.

13. Molitor JA, Ballard DW, Greene WC. Kappa B-specific DNA binding proteins are differentially inhibited by enhancer mutations and biological oxidation. New Biol $1991 ; 3: 987-96$.

14. Matthews JR, Wakasugi N, Virelizier JL, Yodoi J, Hay $\mathrm{RT}$. Thioredoxin regulates the DNA binding activity of NF-KB by reduction of a disulphide bond involving cysteine 62 .

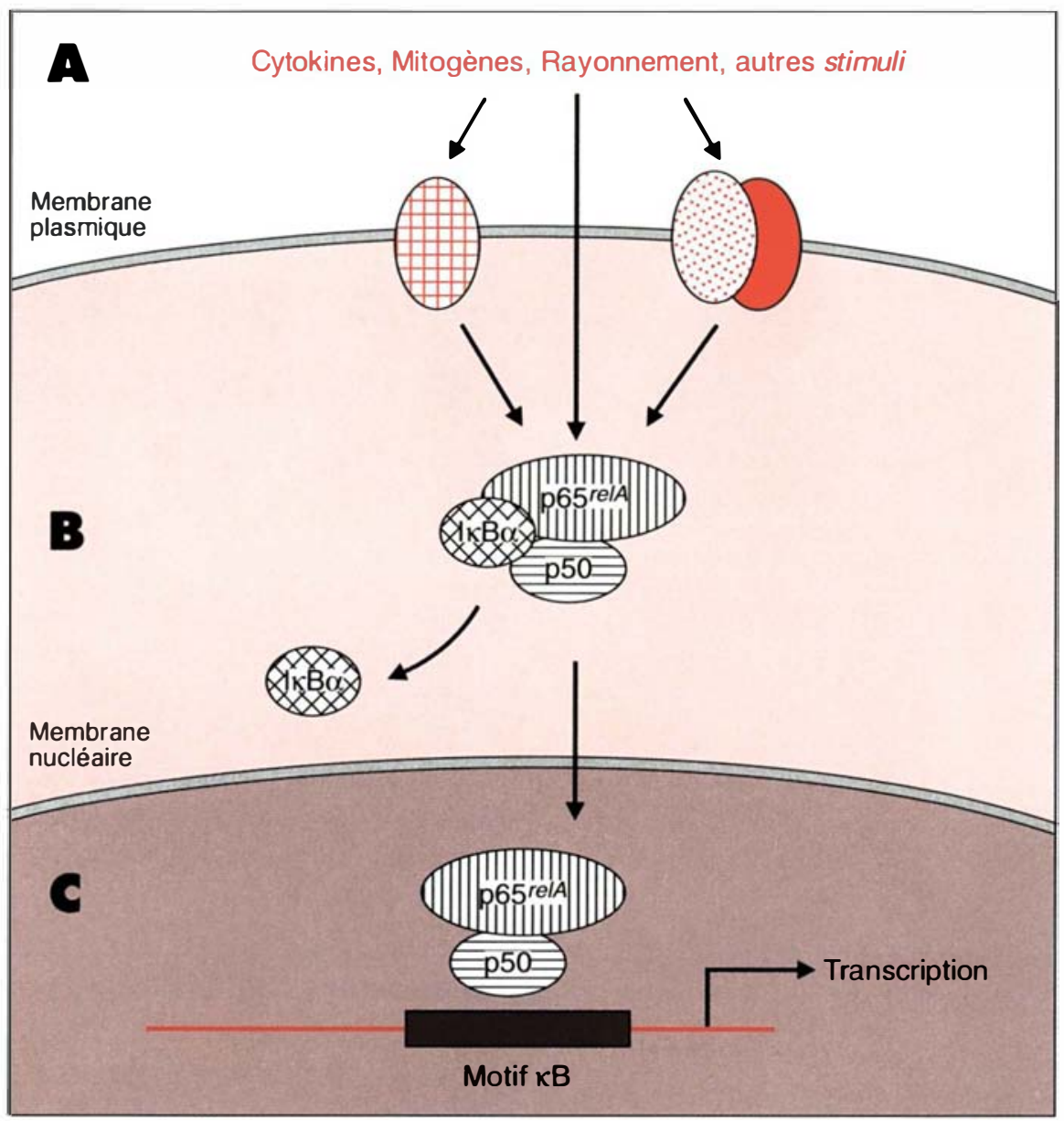

Figure 1. Modèle canonique de l'activation du facteur de transcription NF$\kappa \boldsymbol{B}$. Lors $d^{\prime}$ une stimulation cellulaire $(A)$, divers phénomènes concourent à séparer $1 \kappa B \alpha$ de l'hétérodimère p50/p65 (B), démasquant les signaux de localisation nucléaire (NLS) et permettant sa redistribution vers le noyau où il peut se fixer sur les sites $\kappa B$ accessibles (C).

Figure 2. Membres de la famille Rel/NF- $K B / / \kappa B$ chez les mammifères. Les protéines de la famille Rel/NF- $\kappa B / / \kappa B$ identifiées chez les mammifères sont organisées en domaines. La majorité des gènes correspondants ont été clonés chez l'homme à l'exception de RELB, IкB $\beta$ et IKB $\gamma$ clonés uniquement chez la souris à l'heure actuelle. NRD: motif d'homologie de NF- $\kappa B$, Rel et du morphogène Dorsal de la drosophile; NLS: nuclear localisation signal, signal de localisation nucléaire; $D$ : domaine de dimérisation et de liaison à I'ADN; ANK: motifs d'homologie avec l'ankyrine; $P$ : site potentiel de phosphorylation; GGG : région charnière riche en glycine. Le domaine potentiel d'activation de la transcription est indiqué en bistre. 


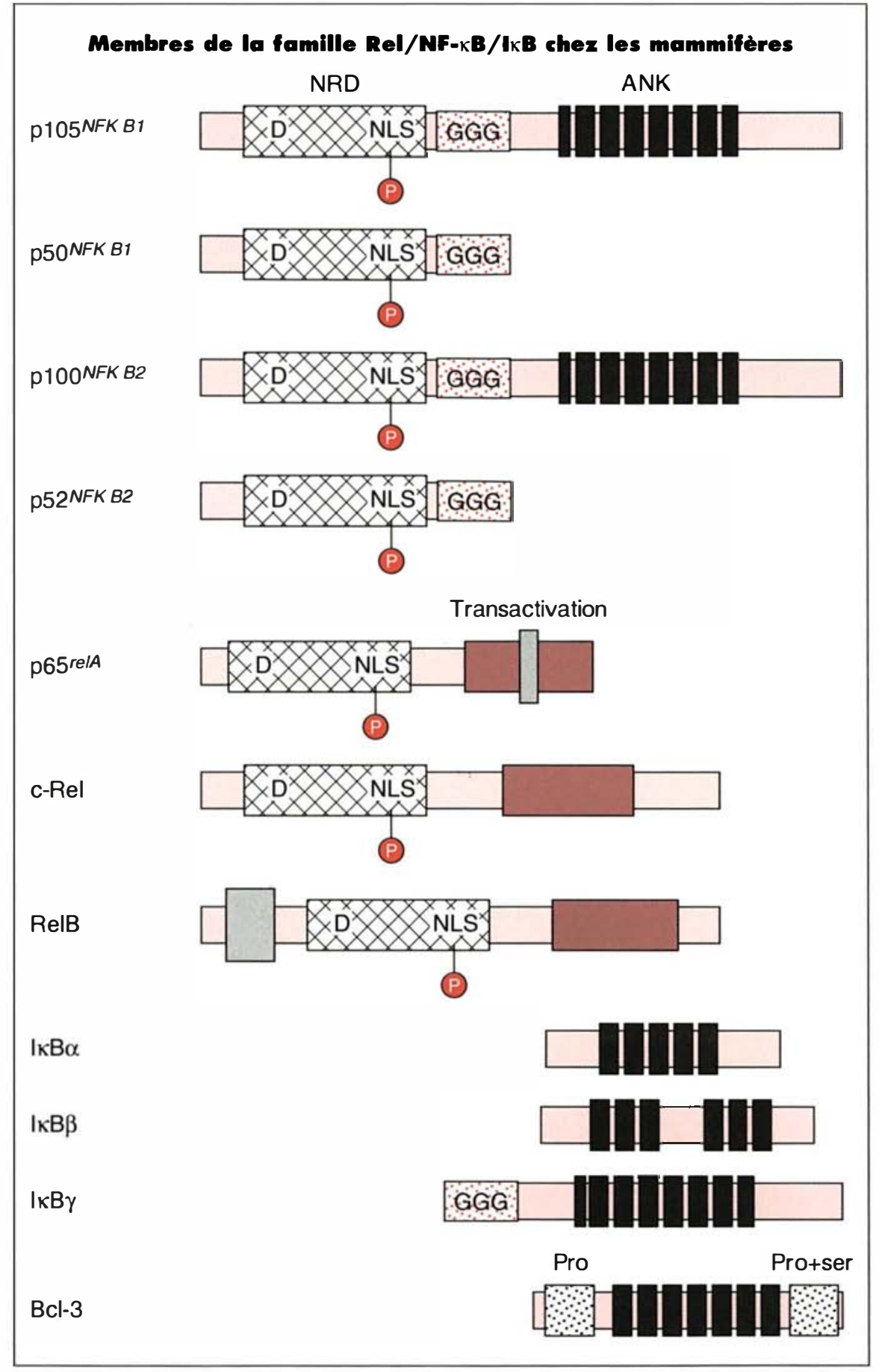



mécanismes d'activation et de régulation du système Rel/NF- $\kappa \mathrm{B}$ sont en réalité plus complexes que ce schéma. Les protéines Rel/NF- $\kappa \mathrm{B}$ et $1 \kappa \mathrm{B}$ constituent en effet une famille de facteurs de transcription (figure 2) dont les gènes respectifs partagent deux régions d'homologie. Brièvement, les sous-unités Rel/NF-kB possèdent une analogie de séquence dans le domaine amino-terminal retrouvée dans le proto-oncogène $\mathrm{c}$-Rel et le morphogène Dorsal de la drosophile (NRD) [1]. Cette région contient le domaine de fixation et de dimérisation (D) ainsi que le signal de localisation nucléaire (NLS) et un site potentiel de phosphorylation $(\mathrm{P})$. La famille $\mathrm{I} \kappa \mathrm{B}$ présente une répétition de motifs ankyrine (ANK) retrouvés dans le produit de plusieurs gènes (ankyrine érythrocytaire, facteurs de transcription, morphogènes...). Des répétitions de motifs ANK, qui interviennent dans les interactions protéine-protéine, sont aussi retrouvées dans le régulateur nucléaire Bcl-3 et dans la partie carboxy-terminale des précurseurs pl05 et pl00 produits respectifs des gènes NFKB1 et NFKB2. L'élément de régulation $\kappa B$, site de fixation spécifique des complexes Rel/NF- $\kappa \mathrm{B}$, a d'abord été identifié au niveau du promoteur des chaînes légères $\kappa$ des immunoglobulines. Des éléments apparentés se retrouvent dans les régions régulatrices de nombreux gènes inductibles, en particulier ceux codant pour des cytokines et leurs récepteurs. La comparaison de ces éléments a permis de définir un site consensus décamérique (5'-GGGANNYYCC-3')* composé de deux hémisites pentamériques qui participent chacun à la reconnaissance et à la fixation des complexes. La diversité des possibilités d'association des membres activateurs et/ou répresseurs par leurs domaines d'homologie (NRD et ANK) et la variabilité de l'affinité des éléments régulateurs $\kappa B$ multiplient de façon spectaculaire les possibilités de modulation du système d'activation Rel/NF-kB (figure 3). Ajoutons enfin qu'il existe de nombreuses similitudes entre le système $\mathrm{Rel} / \mathrm{NF}-\kappa \mathrm{B}$ des mammifères et la

* Oú $N$ est n'importe quel nucléotide et $\gamma$ un nucléotide ì base prymimidigue. 


\section{RÉFÉRENCES}

15. Costello R, Lipcey $\mathrm{C}$, Algarte M, et al. Activation of primary human T-lymphocytes through CD2 plus CD28 adhesion molecules induces long-term nuclear expression of NF-KB. Cell Growth Differ 1993; 4:329-39.

16. Roederer M, Ela SW, Staal FJ, Herzenberg LA. N-acetylcysteine: a new approach to anti-HIV therapy. ADDS Res Hum Retrovir $1992 ; 8: 209-17$.

17. Suzuki YJ, Packer L. Inhibition of NF-KB activation by vitamin $\mathrm{E}$ derivatives. Biochem Biophys Res Commun 1993; 193: 277-83.

18. Schieven GL, Kirihara JM, Myers DE Ledbetter JA, Uckun FM. Reactive oxygen intermediates activate $\mathrm{NF}-\mathrm{KB}$ in a tyrosine kinase-dependent mechanism and in combination with vanadate activate the $p 56^{h k}$ and $5^{59^{\prime n}}$ tyrosine kinases in human lymphocytes. Blood 1993; 82: 1212-20.

19. Menon SD, Qin S, Guy GR, Tan YH. Differential induction of nuclear NF- $\mathrm{KB}$ by protein phosphatase inhibitors in primary and transformed human cells. Requirement for both oxidation and phosphorylation in nuclear translocation. J Biol Chem 1993; 268 : 26805-12.

20. Kanner SB, Kavanagh TJ, Grossmann A, et al. Sulfhydryl oxidation down-regulates Tcell signaling and inhibits tyrosine phosphorylation of phospholipase Cyl. Proc Nat Acad Sci USA 1992; 89: 300-4.

21. Michell RH, Wakelan MJO, Sphingolipid signalling. Curr Biol 1994; 4 : 370-3.

22. Schutze S, Potthoff $K$, Machleidt T, Berkovic D, Wiegmann K, Kronke M. TNF activates NF-KB by phosphatidylcholine-specific phospholipase C-induced «acidic " sphingomyelin breakdown. Cell 1992; 71 : 765-76.

23. Machleidt $T$, Wiegmann $K$, Henkel $T$, Schutze S, Baeuerle PA, Kronke M. Sphingomyelinase activates proteolytic IKB-alpha degradation in a cell-free system. I Biol Chem $1994 ; 269: 13760-5$.

24. Yang Z, Costanzo M, Golde DW, Kolesnick RN. Tumor necrosis factor activation of the sphingomyelin pathway signals nuclear factor kappa B translocation in intact HL-60 cells. I Biol Chem 1993; 268: 20520-3.

25. Dressler KA, Mathias S, Kolesnick RN. Tumor necrosis factor-alpha activates the sphingomyelin signal transduction pathway in a cell-free system. Science 1992; 255: 1715-8.

26. Dobrowsky RT, Kamibayashi C, Mumby MC, Hannun YA. Ceramide activates heterotrimeric protein phosphatase 2A. J Biol Chem 1993; 268: 15523-30.

27. Lenardo M, Siebenlist U. Bcl-3-mediated nuclear regulation of the NF- $\mathrm{KB}$ trans-activa-

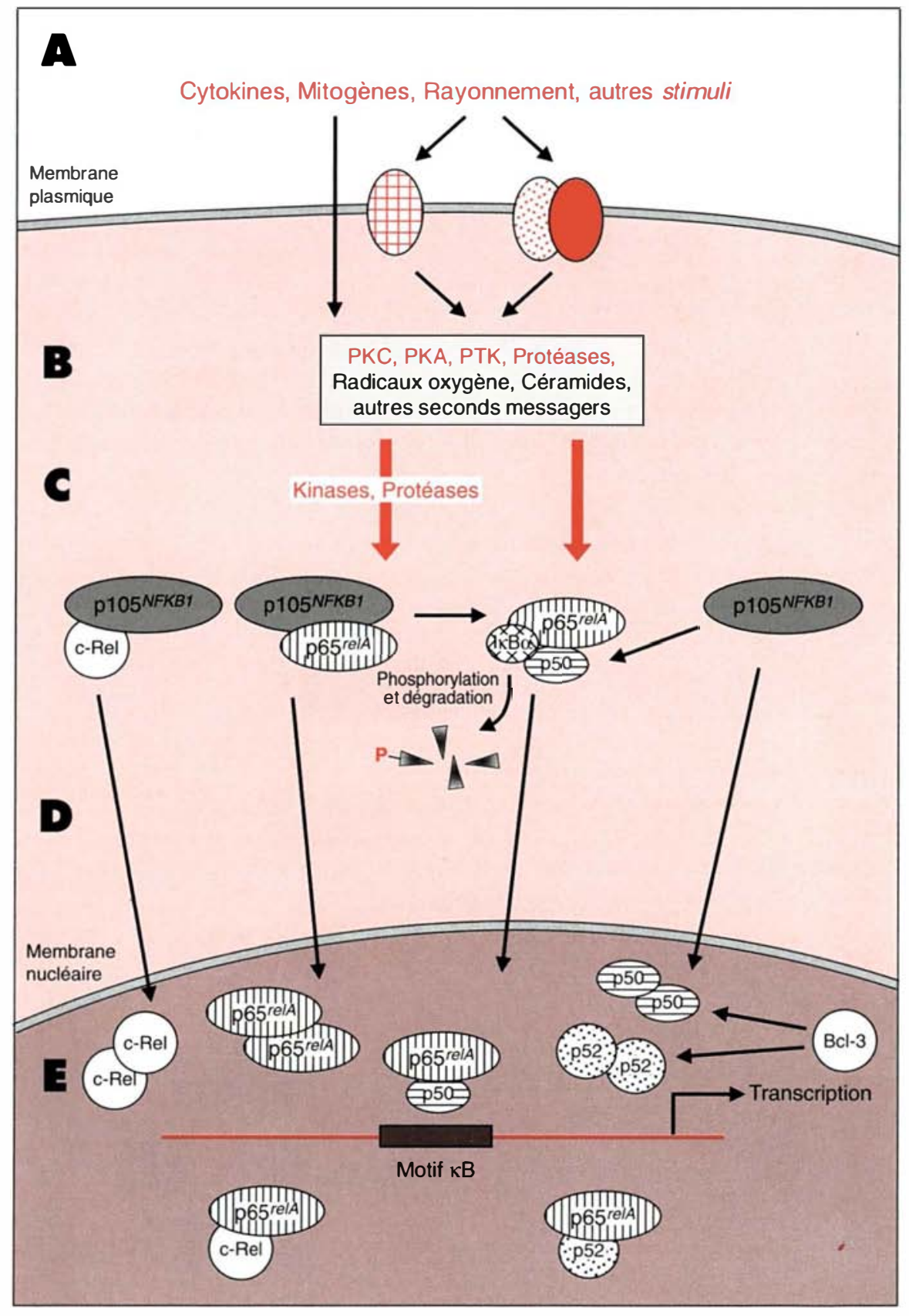

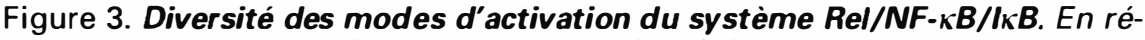
ponse à de nombreux signaux extracellulaires (A) une cascade de messagers secondaires (B) conduit à l'inactivation des sous-unités inhibitrices, à la ma-

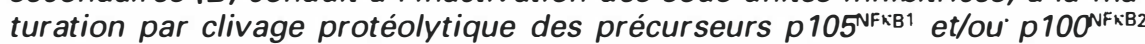
(non représenté dans ce schéma) ainsi qu'à l'activation des sous-unités transactivatrices (C), permettant ainsi la translocation au noyau des complexes $N F-\kappa B$ actifs sur la transcription (D). Dans le noyau (E), ne sont représentées que certaines des combinaisons possibles des membres de la famille Rel/NF. $\kappa B / I \kappa B$ dont les gènes ont été clonés chez l'homme. Les homodimères p50 et p52 dépourvus de domaine de transactivation se comporteraient comme inhibiteurs de la transcription. N'interagissant pas avec $I_{\kappa} B \alpha$, leur activité serait entièrement contrôlée dans le noyau. Le facteur nucléaire $\mathrm{Bcl}$-3 jouerait un rôle de régulateur positif, soit en déstabilisant les homodimères p50, soit en formant des complexes ternaires transactivateurs avec les sous-unités p52 et leur site $\mathrm{\kappa B}[27]$. 
Tableau I

PHOSPHORYLATION CONSTITUTIVE ET INDUITE DES FACTEURS Rel/NF- $\kappa \mathrm{B} / \mathrm{I} \kappa \mathrm{B}$

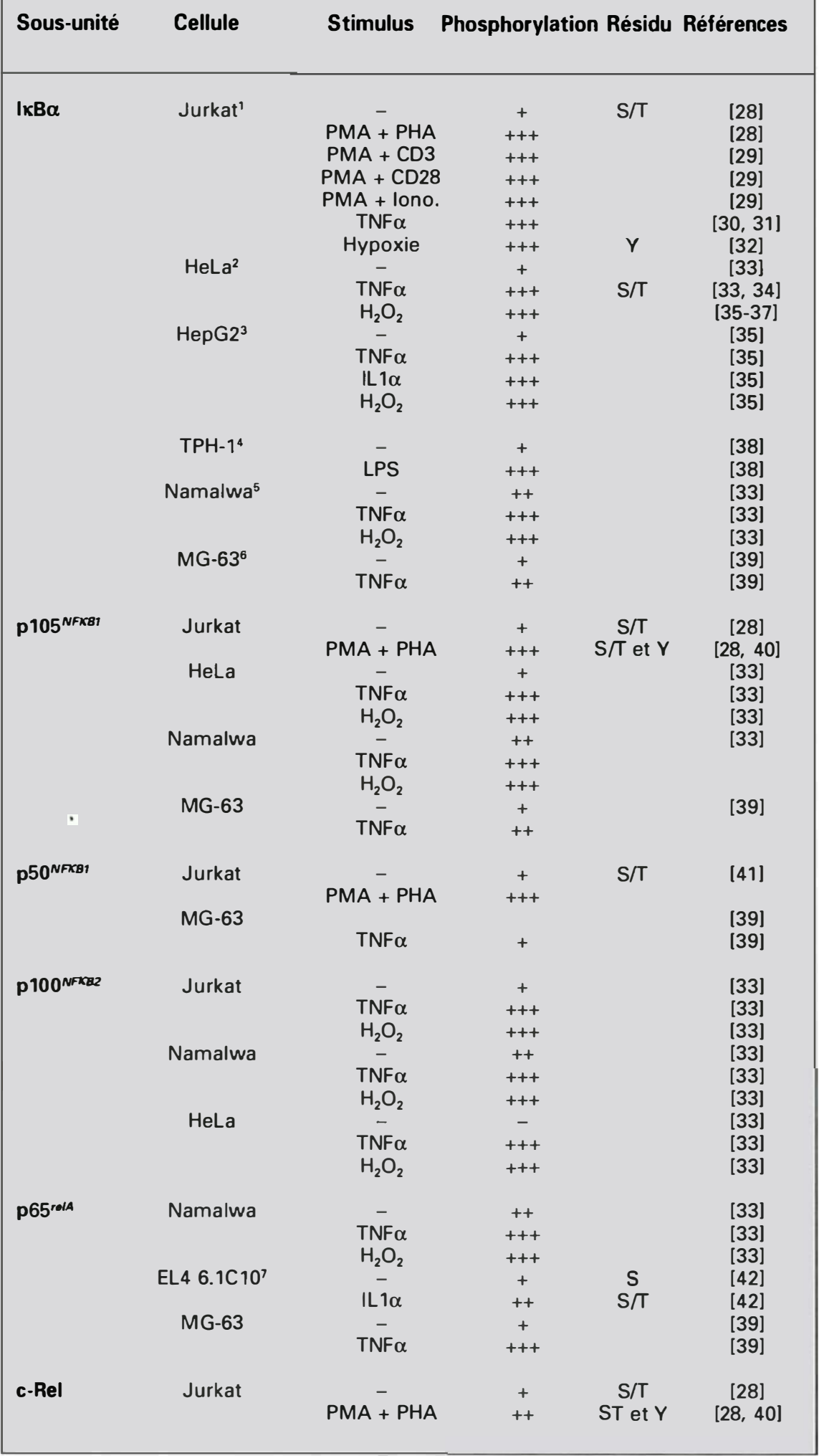

régulation de l'expression des gènes de la différenciation dorsoventrale de l'embiyon de drosophile. Les relations structure/fonction de cette famille ayant été développées dans une revue récente [2], nous avons centré notre présentation sur les mécanismes d'activation du système $\mathrm{Rel} / \mathrm{NF}-\kappa \mathrm{B} / \mathrm{I} \kappa \mathrm{B} \alpha$.

\section{Phosphorylation, déphosphorylation et protéolyse}

La modulation du niveau de phosphorylation des protéines est à l'origine de la régulation de multiples événements cellulaires. Ce phénomène contrôle en particulier l'activité de nombreux facteurs de transcription (par exemple CREB) et/ou d'oncogènes (Jun/Fos, Myc et Myb) ou même d'anti-oncogènes ( 553 , produit du gène de susceptibilité au rétinoblastome). I.es membres de la famille Rel/NF- $\kappa \mathrm{B} / \mathrm{I} \kappa \mathrm{B}$ sont euxmêmes des phosphoprotéines dont la phosphorylation est modulée quantitativement et/ou qualitativement selon le type cellulaire et le stimulus activateur (Tableau I). Dans la majorité des systèmes, on observe une faible phosphorylation basale, corrélée au pouvoir activateur réduit de ces facteurs en l'absence de stimulus d'activation. En revanche, dans les lymphocytes B différenciés où les

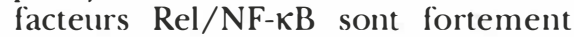
induits à l'état constitutif, la phosphorylation basale est importante.

Seules les phosphorylations in vivo des sous-

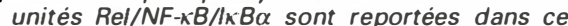
tableau. PMA: phorbol myristyl acétate; PHA phythemagglutinine; CD3: composant invariant du récepteur de l'antigène $T$ comprenant 3 chaines $\gamma, \delta$ et $\varepsilon$ associèes en deux hétérodimères $\gamma \delta$ et $\gamma \varepsilon$ transducteurs; CD28: protéine de $44 \mathrm{kDa}$ présente à la surface de la plupart des lymphocytes $T$ différenciés, impliquée dans les interactions T-B; iono.: ionophore $\mathrm{Ca}^{2+}$ (A23187); TNF: tumor necrosis factor: $\mathrm{H}_{2} \mathrm{O}_{2}$ : peroxyde d'hydrogène; LPS: lipopolysaccharides.' Jurkat: lignée leucémique $T$ humaine, 2 Hela: carcinome épithélioide humain; HepG2: lignée hépatoblastique humaine; ‘ TPH-I: lignee monocytique humaine; ${ }^{5} \mathrm{Namal}-$ wa: lymphome de Burkitt humain: ${ }^{6}$ MG-63: os. wa: lymphome de Burkitt humain;' MG-63: osmurin sécrétant de I'IL2 en réponse à I'IL 1. S: serine, $T$ : thréonine, $Y$ : tyrosine. 


\section{RÉFÉRENCES}

28. Li CCH, Korner M, Ferris DK, Chen EY, Dai RM, Longo DL. NF-KB/Rel family members are physically associated phosphoproteins. Biochem J 1994 ; 303 : 499-506.

29. Alkalay I, Yaron A, Hatzubai A, et al. In vivo stimulation of I kappa B phosphorylation is not sufficient to activate NF-KB. Mol Cell Biol 1995; 15 ; 1294-301.

30. Miyamoto S, Maki M, Schmitt MJ, et al. Tumor necrosis factor alpha-induced phosphorylation of $I K B$ alpha is a signal for its degradation but not dissociation from NFkappa B. Pror Natl Acad Sci USA 1994; 91 : 12740-4.

31. Sun SC, Ganchi PA, Beraud C, Ballard DW, Greene WC. Autoregulation of the NF$\kappa \mathrm{B}$ transactivator RelA (p65) by multiple cytoplasmic inhibitors containing ankyrin motifs. Proc Nall Acad Sci USA 1994; 91 : 1346-50.

32. Koong AC; Chen EY, Ciaccia AJ. Hypoxia causes the activation of nuclear factor $\kappa B$ through the phosphorylation of IKB on tyrosine residues. Cancer Res 1994 ; 54 : 1425 30 .

33. Naumann $\mathbf{M}$, Scheidereit C. Activation of NF-KB in vivo is regulated by multiple 607.

34. Traenckner EBM, Wilk S, Bacuerle PA. A proteasome inhibitor prevents activation of NF-KB and stabilizes a newly phosphorylated form of I kappa B alpha that is still bound to NF-kappa B. EMBO J 1994; 13: $5433-41$.

35. DiDonato JA, Mercurio F, Karin $M$. Phosphorylation of $I \kappa B \alpha$ precedes but is not sufficient for its dissociation from NFкB. Mol Cell Biol 1995; 15: 1302-11.

36. Finco $\mathrm{TS}$, Beg AA, Baldwin AS. Inducible phosphorylation of IkB alpha is not sufficient for its dissociation from NF-KB and is inhibited by protease inhibitors. Proc Nall Acad Sci USA 1994; 91 : 11884-8.

37. Beg AA, Finco TS, Nantermet PV, Baldwin AS, Jr. Tumor necrosis factor and interleukin-l lead to phosphorylation and loss of I $\mathrm{B} \alpha$ : a mechanism for NF-KB activation. Mol Cell Biol 1993; 13: 3301-10.

38. Cordle SR, Donald R, Read MA, Hawiger I. L ipopolysaccharide induces phosphorylation of MAD3 and activation of c-Rel and related $\mathrm{NF}-\mathrm{KB}$ proteins in human monocytic THP-1 cells. J Biol Chem 1993; 268: 11803-10.

39. Neumann M, Tsapos K, Scheppler JA, Ross I, Franza BR, Jr. Identification of complex formation between two intracellular tyrosine kinase substrates: human c-Rel and the p 105 precursor of $\mathrm{p} 50 \mathrm{NF}-\mathrm{KB}$. Oncogene 1992; 7: 2095-104.
Quelle que soit la cellule, la majorité des stimuli induisent une nette élévation du niveau de phosphorylation.

l'impact de la phosphorylation des facteurs sur leur activité inhibitrice ou activatrice a été montré dans un bon nombre de cas (Tableau II). I.e premier indice d'une régulation de l'activité NF-KB par modification de phosphorylation a été obtenu en démontrant que le traitement par la protéine kinase dépendante de I'AMPc (PKA) ou la protéine kinase C (PKC) d'extraits cytosoliques de cellules non stimulées induisait la liaison de ce facteur à l'ADN [3, 4] Un tel résultat suggérait que NF-кB existe dans le cytoplasme de cellules au repos sous une forme inactive qui peut être convertie par un processus de phosphorylation en une forme capable d'être transportée vers le noyau et de se fixer à l'ADN. Cette hypothèse est désormais confortée par plusieurs études qui ont permis d'établir une relation de cause à effet directe entre, d'une part, la phosphorylation de l'inhibiteur I $\kappa \mathrm{B} \alpha$ et son ciblage vers la dégradation par la voie ubiquitine/protéasome [5] et, d'autre part, entre la phosphorylation du précurseur inhibiteur pl 05 et la production de son sous-produit p50. Contrairement à ce qui se passe in vitro, la phosphorylation in vivo d'IK $B \alpha$ n'induit pas, à elle seule, sa dissociation des complexes Rel/NF$\kappa \mathrm{B}$. Ainsi, en l'absence d'une protéolyse subséquente de cet inhibiteur, les complexes demeurent dans le cytoplasme. Le précurseur pl05 paraît réglé in vitro de la même manière que ІкВ $\alpha$ puisqu'il perd, après phosphorylation, sa capacité d'interagir avec les sous-unités p65 ou p50. De plus amples analyses sont néanmoins nécessaires pour établir si, à l'image d'IKB $\alpha$, pl05 (ou pl00) phosphorylé in vivo devient la cible d'un clivage ou d'une dégradation alors qu'il est encore associé à la sous-unité qu'il séquestre. Outre la libération des complexes cytosoliques, différents mécanismes d'activation réglant directement l'activité nucléaire des facteurs Rel/NF-kB dépendraient également de processus de phosphorylation. Ainsi, l'activité des deux régulateurs $I \kappa B$ nucléaires, $\mathrm{I} \kappa \mathrm{B} \gamma$ et $\mathrm{BCl}-3$, apparaît-elle modulée in vitro par phosphorylation. En outre, la phosphorylation de la sousunité transactivatrice p65 ainsi que celle de la sous-unité p50 induisent,

\begin{tabular}{|c|c|c|}
\hline \multicolumn{3}{|c|}{$\begin{array}{l}\text { ACTIVATION DÉ NF-кB PAR PHOSPHORYLATION } \\
\text { DES SOUS-UNITÉS ACTIVATRICES ET INHIBITRICES }\end{array}$} \\
\hline Cible & Mécanisme induit & Références \\
\hline $\mid \kappa B \alpha$ & Ciblage vers la dégradation & $\begin{array}{l}{[4,29-31,33-37,} \\
39,43,44]\end{array}$ \\
\hline IKBy & $\begin{array}{l}\text { Abrogation de l'inhibition de la } \\
\text { liaison à l'ADN des homodimères } \\
\text { p50 et c-Rel }\end{array}$ & [45] \\
\hline Bcl-3 & $\begin{array}{l}\text { Déstabilisation de la liaison à l'ADN } \\
\text { des homodimères p50 }\end{array}$ & {$[46]$} \\
\hline p105 NFKB1 & $\begin{array}{l}\text { Inhibition de l'association p105/p65 } \\
\text { et } p 105 / p 50 \\
\text { production de } p 50\end{array}$ & $\begin{array}{l}{[33,47]} \\
{[33,39]}\end{array}$ \\
\hline p50 NFKB1 & $\begin{array}{l}\text { Stabilisation de la liaison à l'ADN } \\
\text { des homodimères p50 et des } \\
\text { hétérodimères p50/p65 }\end{array}$ & [41] \\
\hline p65 $5^{\text {Re/A }}$ & $\begin{array}{l}\text { Stabilisation de la liaison à l'ADN } \\
\text { des homodimères p } 65 \text { et des } \\
\text { hétérodimères p } 50 / p 65\end{array}$ & [33] \\
\hline
\end{tabular}


in vitro également, une stabilisation de la liaison de ces sous-unités à l'ADN.

De multiples travaux s'orientent vers l'élucidation des protéine kinases cytosoliques et/ou nucléaires directement impliquées dans la phosphorylation des facteurs Rel/NF- $\kappa \mathrm{B} / \mathrm{I} \kappa \mathrm{B}$. Diverses analyses de phosphoaminoacides ont montré que des sérines et des thréonines sont phosphorylées in vivo sur toutes les sous-unités (Tableau I), indiquant l'intervention d'une ou plusieurs sérine/thréonine kinase(s). Différents arguments sont en faveur d'un rôle de la PKA. Tout d'abord, chacune des sous-unités Rel/NF-kB possède, dans son domaine d'homologie (NRI)), un site consensus (Arg-Arg-Pro-Ser) de phosphorylation par la PKA (figure 2). Plus indirectement, NF- $\kappa \mathrm{B}$ peut, dans certains contextes cellulaires, être activé par des augmentations du taux d'AMPc $[3,6]$. Dans le thymome murin EL4, en revanche, l'induction de la PKA inhibe l'activité NF-kB [7]. Ce dernier effet de la PKA étant différé, il n'est pas impossible que cette kinase participe à la fois à l'activation précoce du facteur ainsi qu'à son autorégulation négative plus tardive. Puisque la relocalisation nucléaire de la sous-unité catalytique de la PKA est également différée, l'activité inhibitrice de cette kinase pourrait s'exercer dans le noyau. Deux isoenzymes

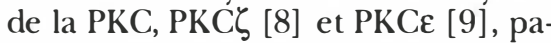
raissent également impliquées dans l'activation in vivo de NF-KB. Cependant, tout comme pour la PKA, les cibles directes de ces kinases restent à définir. Plusieurs autres sérine/ thréonine kinases, inconnues à l'exception de Raf-l et de la protéine kinase ARN-dépendante (PKR), ont, en revanche, été directement copurifiées avec des protéines Rel/NF$\kappa \mathrm{B} / \mathrm{I} \kappa \mathrm{B}$ à partir d'extraits cytosoliques ou nucléaires de cellules activées (Tableau III).

La phosphorylation sur tyrosine de différentes sous-unités a également été démontrée après activation cellulaire (Tableau I), révélant la participation d'une ou plusieurs activités tyrosine kinase(s). Etant donné la pluralité des sérine/thréonine et tyrosine kinases candidates, il est vraisemblable que les différents mécanismes d'acti-

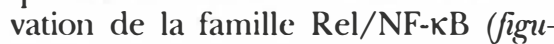
re 3 et Tableau II) mettent en synergie $\mathrm{m} / \mathrm{s} n^{\circ} 7$, wol. 11, juillet 95

Tableau III

SÉRINE/THRÉONINE KINASES DIRECTEMENT

ASSOCIÉES AUX FACTEURS Rel/NF- $\kappa B / / \kappa B$

\begin{tabular}{|c|c|c|c|c|}
\hline Cible & Kinase & $\begin{array}{c}\text { Localisation } \\
\text { cellulaire }\end{array}$ & $\underset{\text { (kDa) }}{\text { PM }}$ & Références \\
\hline $\mid \mathbf{I} B \alpha$ & 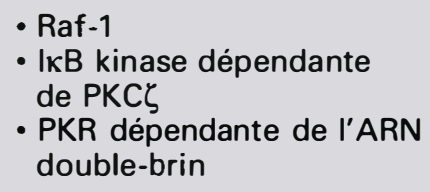 & $\begin{array}{l}\text { cytosol } \\
\text { cytosol }\end{array}$ & $\begin{array}{l}74 \\
50 \\
68\end{array}$ & $\begin{array}{l}{[48]} \\
{[49]} \\
{[50]}\end{array}$ \\
\hline p50 NFKB1 & NF- $k B$ kinase & cytosol & 43 & [51] \\
\hline p $65^{\text {RelA }}$ & $\begin{array}{l}\text { - NF-kB kinase } \\
\text { - p65 kinase }\end{array}$ & $\begin{array}{l}\text { cytosol } \\
\text { noyau }\end{array}$ & $\begin{array}{c}43 \\
?\end{array}$ & $\begin{array}{l}{[51]} \\
{[42]}\end{array}$ \\
\hline
\end{tabular}

Raf-1: sérine/thréonine kinase réglée par la petite protéine $G$ Ras, qui exerce son activité en amont de la cascade des kinases activées par les mitogènes (MEK et MAPK): PKCK: isotype de la PKC insensible au 12-O-tétradécanoyl phorbol 13-acétate (TPA); PKR: sérine/thréonine kinase, réglée par l'ARN double brin, qui phosphoryle la sous-unité $\alpha$ du facteur d'initiation 2 (elF-2).

différentes combinaisons de kinases constitutives ou induites.

Plusieurs travaux évoquent l'implication de protéine kinases et de phosphatases agissant en amont des enzymes décrites ci-clessus. De plus amples analyses sont donc nécessaires pour établir la cascade d'événements de phosphorylation conduisant à l'activation des facteurs Rel/NF-кB. Cette activation chez les mammifères, ainsi que celle de la protéine Dorsal chez la drosophile, sont apparemment contrôlées par des mécanismes remarquablement conservés, en accord avec les fortes analogies structurales des membres de cette famille. Chez la drosophile, l'activation du récepteur transmembranaire Toll déclenche une cascade de signaux intracellulaires mettant en jeu les sérine/thréonine kinases Pelle et Tube et conduisant à la libération du facteur de transcription Dorsal de son inhibiteur Cactus [10]. I.'élucidation de ce modèle sur des bases biochimiques et génétiques devrait accélérer la compréhension des mécanismes homologues chez les mammifères.

\section{Oxydoréduction}

Les phénomènes d'oxydoréduction semblent jouer un rôle important dans l'activation de NF- $\kappa \mathrm{B}$ en permettant son transport nucléaire, probablement grâce à l'inactivation de l'inhibiteur $I \kappa B \alpha$ [11]. De façon directe, l'action oxydante puissante de l'eau oxygénée induit l'activation de NF- $\kappa$ B dans une lignée leucémique $T$ Jurkat. De même, la stimulation de cellules Jurkat par le TNF $\alpha$ ou le PMA induit une chute des thiols intracellulaires, inhibée par l'adjonction de $\mathrm{N}$-acétyl-cystéine (NAC), puissant neutralisant des radicaux libres. Le NAC inhibe l'activation de NF- $\kappa B$ et l'expression d'un vecteur à gène indicateur sous le contrôle d'un triple motif $\kappa \mathrm{B}$. Des résultats comparables ont été obtenus avec un autre agent neutralisant de radicaux libres, le pyrrolidine dithiocarbamate (PITC). L'inhibition in vitro de la

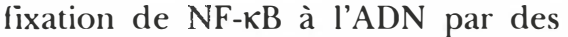
agents oxydants $[12,13]$ suggère que les radicaux libres peuvent agir également comme des régulateurs négatifs de l'activation. En accord avec cette hypothèse, l'oxydation de la cystéine en position 62 de p50 $0^{N F \times 31}$ inhibe la liaison de cette sous-unité à l'ADN [14]. Le paradoxe entre ces effets contradictoires n'est sans doute qu'apparent. Dans le premier groupe d'études, des cellules intactes sont soumises à des agents neutralisants de radicaux libres alors que, dans le second, il s'agit de travaux effectués dans un système acellulaire (agents oxydants ou antioxydants ajoutés $a$ posteriori dans les extraits cellulaires). L'oxydation peut aussi bien induire le détachement de p50/p65 de son 


\section{RÉFÉRENCES}

40. Mellits KH, Hay RT, Goodbourn S. Proteolytic degradation of MAD3 $(\mathrm{I} \kappa \mathrm{B} \alpha)$ and enhanced processing of the NF-KB precursor p105 are obligatory steps in the activation of NF-кB. Nucleic Acids Res 1993; 21 : 5059-66.

41. Li CCH, Dai RM, Chen E, Longo DL. Phosphorylation of NF-KBl p50 is involved in NF-KB activation and stable DNA binding. J Biol Chem 1994; 269: 30089-92.

42. Ostrowski J, Sims JE, Sibley CH, el al. A serine/threonine kinase activity is closely associated with a $65-\mathrm{kDa}$ phosphoprotein specifically recognized by the $\mathrm{KB}$ enhancer element. I Biol Chem 1991 ; 266: 12722-33.

43. Kerr LD, Inoue J, Davis N, et al. The relassociated pp40 protein prevents DNA binding of Rel and NF-KB : relationship with ding of Rel and NF- $K B$ : relationship with Genes Dev $1991 ; 5$ : 1464-76.

44. Henkel T, Machleidt T, Alkalay I, Kronke M, Ben-Neriah Y, Baeuerle PA. Rapid proteolysis of $I \kappa B \alpha$ is necessary for activation of transcription factor NF-KB. Nature $1993 ; 365$ : $182-5$.

45. Gerondakis $\mathrm{S}$, Morrice $\mathrm{N}$, Richardson IB, Wettenhall R, Fecondo J, Grumont RJ The activity of a 70 kilodalton I $\mathrm{KB}$ molecule identical to the carboxyl terminus of the p105 NF- $\mathrm{KB}$ precursor is modulated by protein kinase A. Cell Growth Differ 1993; 4:617 27.

46. Nolan GP, Fujita T, Bhatia $\mathrm{K}$, et al. The $b c l-3$ proto-oncogene encodes a nuclear I $\mathrm{KB}$ like molecule that preferentially interacts with NF-KB p50 and p52 in a phosphorylation-dependent manner. Mol Cell Biol 1993; 13: 3557-66.

47. Rice NR, Mackichan ML, Israel A. The precursor of NF-KB p50 has IKB-like functions. Cell 1992; 71 : 243-53.

48. Li S, Sedivy JM. Raf-1 protein kinase activates the NF-KB transcription factor by dissociating the cytoplasmic NF-KB-IKB complex. Proc Natl Acad Sci USA 1993; 90: 9247-51.

49. Diaz-Meco MT, Dominguez I, Sanz L, et al. zeta PKC induces phosphorylation and inactivation of I kappa B-alpha in vitro. EMBO J 1994; 13: 2842-8.

50. Kumar A, Haque J, Lacoste J, Hiscott J, Williams BR. Double-stranded RNA-dependent protein kinase activates transcription factor NF-kB by phosphorylating IKB. Proc Nall Acad Sci USA 1994; 91 : 6288-92.

51. Hayashi T, Sekine T, Okamoto T. Identification of a new serine kinase that activates NF-kB by direct phosphorylation. I inhibiteur cytoplasmique que de son site de fixation à l'ADN. Des expériences complémentaires sont donc nécessaires pour vérifier l'intervention différentielle des mécanismes d'oxydoréduction en fonction du compartiment cellulaire. En accord avec cette hypothèse, les variations de potentiel redox in vivo ont surtout lieu au niveau cytosolique, ce qui permettrait un rôle dans le transport nucléaire sans inhiber la fixation à l'ADN de NF- $\kappa B$.

Dans le lymphocyte T humain purifié du sang périphérique, le PDTC. est un puissant inhibiteur de l'activation des fonctions lymphocytaires et du transport nucléaire de NF- $\mathrm{BB}$ par de nombreux stimuli [15], soulignant l'importance des variations du potentiel redox dans la physiologie de la cellule T. L'importance de ce phénomène dépasse le cadre de la physiologie normale puisque la dérégulation du potentiel redox semble impliquée dans certaines affections virales telles que l'infection par le virus HTI.V-l ou le VIH. Dans ce dernier cas, des taux de glutathion diminués sont observés dans les lymphocytes de patients infectés. Or le glutathion est un des principaux neutralisants de radicaux oxygène libres de la cellule, ce qui suggère la participation d'un état pro-oxydant dans cette maladie. De même, un autre agent réducteur cellulaire, la thioredoxine, a été impliqué dans la régulation redox de $\mathrm{NF}-\kappa \mathrm{B}$ [14]. I.e VIH comporte dans sa région de régulation LTR deux motifs $\kappa \mathrm{B}$ : les phénomènes d'oxydoréduction pourraient donc stimuler la réplication virale indirectement par l'activation de $\mathrm{NF}-\kappa \mathrm{B}$. Différents groupes ont proposé l'utilisation thérapeutique d'agents neutralisants des radicaux libres tels que les dérivés de la cystéine et de la vitamine E sur la base de leurs effets inhibiteurs sur différents modèles cellulaires [16, 17]. Cependant, les essais cliniques d'agents antioxydants n'ont pas pour l'instant domné d'effet probant. Si les phénomènes d'oxydoréduction, impliqués à plusieurs étapes du métabolisme cellulaire participent à l'activation de NF- $\kappa \mathrm{B}$, le mécanisme exact demeure inconnu $\left(\mathrm{m} / \mathrm{s} n^{\circ} 3\right.$, vol. 11, p. 467) (action directe, implication de molécules intermédiaires...).

\section{Oxydoréduction et phosphorylation}

Un autre niveau de régulation provient de l'intrication des réactions d'oxydoréduction et de phosphorylation. Récemment, un lien a été établi entre l'activation de NF- $\kappa \mathrm{B}$ par les radicaux oxygènes et l'activité tyrosine kinase de p56 trk et p59/rn [18]. En outre, l'activation nucléaire de NF- $\kappa \mathrm{B}$ a été mise en évidence par l'action combinée d'agents pro-oxydants tels que le peroxyde d'hydrogène ou le buthionine sulfoximine et d'inhibiteurs des phosphoséryl/thréonyl phosphatases dans diverses lignées humaines primaires et transformées [19]. Dans ce système, la coopération entre phosphorylation et radicaux oxydants pourrait s'exercer par l'inactivation directe de $I \kappa B \alpha$. Enfin, dans des lymphocytes $T$, un état prooxydant puissant diminue la phosphorylation des tyrosines de la phospholipase C $\gamma \mathrm{l}$ (PLC $\gamma \mathrm{l}$ ) et inhibe l'activité de cette enzyme [20]. La PL.C. activant la PKC par l'intermédiaire du diacyl glycérol (DAG), une oxydation forte pourrait inhiber l'induction de cette kinase. Néanmoins, une oxydation modérée plus proche des conditions physiologiques augmente l'activation de la PI.C $\gamma$ l et suggère ainsi que les phénomènes d'oxydation constituent une étape d'activation en amont des phosphorylations dans la cascade des seconds messagers.

\section{La voie des sphingomyélines}

La voie des sphingomyélines suscite un intérêt grandissant comme second messager de la transduction du signal de nombreux récepteurs par son double rôle potentiel d'effecteur positif et/ou négatif de la prolifération et de la différenciation cellulaire [21]. L'activation de la PLC et la production de DAG dans le lymphocyte $T$ aboutissent à d'autres voies métaboliques que les phosphorylations. En effet, la fixation du TNF $\alpha$ à son récepteur induit une activité phosphatidylcholine-phospholipase (') et aboutit à la production de DAG qui active une sphingomyélinase [22]. La sphingomyéline transformée en céramide, molécule très hydrophobe, activerait NF- $\kappa \mathrm{B}$ en agissant sur $\mathrm{I} \kappa \mathrm{B} \alpha$. 
Le mécanisme de cette dernière étape ferait intervenir une protéase activée par le céramide [23]. Fn effet, l'addition de sphingomyélinase ou de céramide dans un essai acellulaire active la dégradation protéolytique d'I $\mathrm{B} \alpha$. Cet effet pourrait s'exercer directement sur l'inhibiteur [24] ou bien, de manière non exclusive, par l'intermédiaire de radicaux oxygène, d'une kinase spécifique [25] ou encore d'une phosphatase cytosolique intervenant dans le rétrocontrôle de l'activité kinase [26].

\section{Conclusion: régulation du système Rel/NF-KB}

En conclusion, la phosphorylation de I $\kappa \mathrm{B} \alpha$ dépendante de la $\mathrm{PKC}$ décrite à l'origine n'est pas la seule voie d'activation possible de NF- $\kappa$ B. Les différentes sous-unités de la famille Rel/NF- $\kappa \mathrm{B} / \mathrm{I} \kappa \mathrm{B}$ peuvent être affectées par des mécanismes multiples associant les phénomènes de phosphorylation/déphosphorylation, les variations de potentiel redox et la protéolyse. Ces trois grands domaines du métabolisme cellulaire apparaissent intimement intriqués, chacune de leur combinaison permettant une réponse adaptée aux multiples stimuli extracellulaires. Cependant, la régulation de ce systè- me ne se limite pas à la translocation nucléaire des complexes Rel/NF- $\kappa \mathrm{B}$ actifs sur la transcription. Il existe en effet d'autres possibilités de contrôle, de l'autorégulation de la transcription des différentes sous-unités à la modulation de l'effet transcriptionnel de NF- $K B$ au niveau des séquences régulatrices des gènes cibles par l'interaction avec d'autres facteurs de transcription

\section{Remerciements}

L.es auteurs remercient le Docteur Alain Israēl pour ses conseils et commentaires ainsi que l'Association pour la Recherche sur le Cancer et le Comité des Bouches du-Rhône de la Ligue Nationale contre le Cancer pour leur soutien financier.

\section{TIRÉS À PART}

J. lmbert.

\section{Summary}

Rel/NF-KB transcription factor: activation and regulation

The family of Rel/NF- $\kappa \mathrm{B}$ transcription factors is involved in the activation of many viral and cellular genes, notably immune response genes coding for cytokines and cytokine receptors. A constitutive DNA-binding activity is detected in some cells such as mature B lymphocytes or monocytes. Nonetheless, in most cell types Rel/NF- $\mathrm{KB}$ nuclear translocation and DNAbinding are inducible by various stimuli such as hormones, cytokines, bacterial and viral infections, lipopolysaccharides, phorbol esters, free oxygen radicals or UVradiations. This nuclear translocation from inactive cytosolic stores involves many non-mutually exclusive mechanisms, allowing a fine tuning of Rel/NF- $\kappa \mathrm{B}$ activation depending on stimuli and cell types. Based upon their adaptable modu-

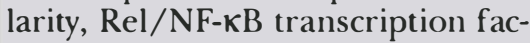
tors appear as a major system for rapid host defense against a wide variety of pathogenic and stress agents including inflammatory phenomena. 